\title{
Produção científica no contexto psicanalítico (2002-2009)
}

\author{
Rosane Zétola Lustoza ${ }^{1}$ - Universidade Estadual de Londrina, Londrina, Brasil \\ Katya Luciane de Oliveira - Universidade Estadual de Londrina, Londrina, Brasil \\ Bianca Novaes de Mello - Pontifícia - Universidade Católica do Rio de Janeiro, Rio de Janeiro, Brasil
}

\begin{abstract}
Resumo
Esta pesquisa teve por objetivo analisar a produção científica no contexto psicanalítico em 229 artigos (2002 a 2009), seis periódicos científicos indexados. A análise baseou-se em alguns critérios da metaciência, a saber, autoria, temática, discurso e análise dos tipos de recursos. Os resultados evidenciaram que em alguns periódicos há maior concentração de publicações sobre a temática, acentuada nos últimos anos. A participação feminina foi predominante na autoria dos artigos, e detectou-se ampla diversificação nos propósitos e contextos nos quais a psicanálise tem sido aplicada. Conclui-se que a produção divulgada no período revela o amadurecimento da área, mas aponta para alguns aspectos que poderão ser mais explorados em futuras publicações. Sugere-se que outros estudos similares sejam realizados, visando monitorar a expansão da área no Brasil. Palavras-chave: Produção científica, Publicação científica, Metaciência, Psicanálise.
\end{abstract}

Scientific production in the psychoanalytic context (2002-2009)

\begin{abstract}
This paper analyzes the scientific production in the psychoanalytic context published in 229 articles (2002 to 2009) from six scientific indexed periodicals. The analysis was carried out according to some metascientific criteria such as: authorship, thematic, discourse, and exam of the different types of resources. Results evinced not only a greater concentration of publications regarding psychoanalysis in certain journals, but also an increase of these types of studies recently. It was also found that most of the authors were females and the psychoanalysis were used both in a variety of contexts and with diverse purposes. It was concluded that the divulged production in the period reveals the matureness of the area, but points out some aspects that might be explored in future publications. It is recommended that similar studies be conducted in order to better monitor the expanding area in Brazil.

Keywords: Scientific production, Scientific publication, Metascience, Psychoanalysis.
\end{abstract}

\section{Contextos da produção científica}

Autores como Barbosa e Moreira (2009), Oliveira Filho, Hochman e Ferreira (2005), Souza Filho, Belo e Gouveia (2006) e Yamamoto, Souza e Yamamoto (1999) apontam que são de suma importância pesquisas que avaliam a produção científica no âmbito nacional. Estudos dessa natureza permitem que se tenha um panorama das políticas de gestão científica vigentes no cenário nacional, o que possibilita compreender a disseminação dos conhecimentos científicos.

Os veículos de divulgação científica, em especial os periódicos, dão visibilidade ao conhecimento das mais diversas áreas do saber. Soares, Victoria, Cavalieri e Bottino (2006) esclarecem que os periódicos científicos podem ser caracterizados como meios de divulgação de investigações e teorias que resultam do emprego da metodologia científica. Acrescentam que tanto os artigos científicos como os

\footnotetext{
${ }^{1}$ Endereço para correspondência:

Universidade Estadual de Londrina - Depto de Psicologia e Psicanálise

Rodovia Celso Garcia Cid, Km 380 - Caixa Postal 6001 - 86051980 - Londrina - PR

E-mail: rosanelustoza@uel.br; katya@uel.br
}

provenientes das revistas populares são disponibilizados on-line. Destarte, os periódicos científicos podem ser acessados especialmente em bases de dados com reconhecida credibilidade científica. Dessa forma, periódicos indexados em bases de dados de relevância científica apresentam fonte de informação segura (Berberian, Krüger, Guarinello \& Massi, 2009). Essa informação é disseminada de forma mais adequada, quando está disponível em sua forma integral nessas bases de dados.

Pode-se dizer que atualmente há maior facilidade para o acesso à informação científica, sobretudo quando se considera a Biblioteca Virtual em Psicologia - BVS-PSI e o PePSIC. Há que se citar ainda as bases como SciELO, PsycInfo (American Psychological Association), LILACS e MEDLINE (National Library of Medecine) (Costa, 2006; Witter, 2005). O Institute for Scientific Informaticon (ISI) e o Scopus também oferecem parâmetros de indexação, sendo, contudo, mais restritos, o que aumenta a qualidade dos periódicos que conseguem atingir o rigor exigido por eles.

Costa e Yamamoto (2008) apontam que houve um crescimento dos periódicos de psicologia, estando, somente no Index Psi Periódicos, aproximadamente 160 revistas científicas brasileiras na área de Psicologia e correlatas. Observam ainda que o Portal de 
Periódicos CAPES agrega aproximadamente 800. Sob essa perspectiva, ressalta-se a importância de mecanismos de avaliação que permitam a qualificação dos periódicos que se propõem a disseminar o conhecimento científico.

Um mecanismo importante de regulação da qualidade da informação científica é oferecido pela CAPES, por meio do Qualis. Trata-se de uma classificação que abrange os periódicos de divulgação científica de todas as áreas. Em síntese, pode-se dizer que os veículos são indexados de acordo com seu fator de impacto e base de dados no qual estão indexados. Assim sendo, a classificação A $(1,2)$ e B (1-5) somente se aplicará aos periódicos com indexação em alguma base de dados, que atendam ainda aos critérios de consistência em sua publicação, sistema de avaliação pelos pares (avaliação às cegas) e corpo editorial formalizado. Os periódicos que não atendem esses critérios são classificados como C. Todavia, Costa e Yamamoto (2008) consideram que, em face da necessidade crescente da produtividade, exigência esta que atinge todos os programas de pós-graduação, muitas vezes a produção científica deixa a desejar, condicionada ao cumprimento de demandas que primam pela quantidade e não tanto pelo conteúdo dessa produção. Portanto, os autores questionam a necessidade de se discriminar entre o conhecimento produzido e a qualidade dessa informação.

Posto isso, parece plausível avaliar a produção científica dos artigos publicados nesses periódicos. Pondera-se que a análise dos artigos permite, ainda que não em sua totalidade, um balizamento da qualidade da informação prestada, especialmente quando se elegem critérios de meta-análise (Witter, 1999; 2005), que possibilitam a classificação da informação prestada. Investigações que focam a produção científica permitem uma leitura crítica das publicações de uma determinada área (Yamamoto \& cols., 2002), o que promove o levantamento de hipóteses sobre a disseminação do conhecimento. Trabalhos sobre produção científica possuem, portanto, grande relevância, pois fornecem um mapeamento das contribuições, necessidades e déficits da produção em uma determinada área, que no caso da presente pesquisa é a psicanálise.

\section{Considerações sobre a pesquisa em psicanálise}

Apesar da intenção explícita de Freud em incluir a psicanálise como um ramo das ciências naturais, os intérpretes de sua obra discutem até hoje qual o modo mais conveniente de conceber seu estatuto epistemológico. Isso explica por que é possível encontrar nos artigos dessa área metodologias não só diferentes, como algumas vezes até mesmo incompatíveis entre si (Freire \& Lustoza, 2005).

Há perspectivas que tratam a psicanálise como uma ciência natural, procurando submetê-la aos parâmetros de validação característicos daquele paradigma. Como representantes dessa corrente podese citar a ego psychology norte-americana e mais recentemente a neuropsicanálise. Esse ponto de vista considera indispensável uma busca de comprovação empírica dos enunciados freudianos, através do emprego de instrumentos que validem as interpretações analíticas por meios independentes, exteriores à experiência clínica. Busca-se ainda uma aliança com as disciplinas biológicas, particularmente as neurociências e a genética, a fim de conferir um maior rigor aos achados freudianos, conforme aponta Cheniaux (2006).

Contudo, há estudiosos da psicanálise que rejeitam a sua pertinência ao programa naturalista, ressaltando ser a experiência analítica algo que se passa inteiramente no registro da significação e/ou da linguagem. Como relata Monzani (1989), tais teóricos consideram que as abordagens objetivistas eliminam justamente aquilo que haveria de mais importante: a experiência da significação pelo sujeito. A legitimidade das asserções da psicanálise deveria ser examinada a partir de outros referenciais metodológicos. A pesquisa, nesse caso, mostraria uma afinidade maior com os procedimentos característicos das ciências humanas e sociais, tais como a história, a antropologia, a sociologia e a linguística (Lustoza, 2007).

Outros autores, como Simanke (2009), apontam ainda a necessidade de ultrapassar a tradicional dicotomia naturalismo versus antinaturalismo, uma vez que as ciências naturais estariam avançando sobre áreas de conhecimento tradicionalmente reservadas às humanidades. $\mathrm{O}$ autor cita o exemplo da sociobiologia na sua relação com as ciências sociais e das neurociências com relação às ciências da mente.

Outro aspecto a ser considerado é que a diversidade do campo psicanalítico não incide apenas sobre os modos de validação de suas proposições. Sendo a obra freudiana um pensamento em permanente movimento (Monzani, 1989), notável por sua riqueza e polifonia, compreende-se que cada intérprete, estribado em diferentes interesses $\mathrm{e}$ interpretações, possa valorizar mais uma faceta do que outra - o que acarreta distintos pontos de vista sobre a natureza do tratamento. Apresentam-se então as escolas de psicanálise, que desenvolveram posteriormente aparatos conceituais e tipos de intervenção próprios. Elas exibem diferenças em relação às concepções nosológicas, às técnicas 
utilizadas e aos objetivos do tratamento. Dentre elas destacam-se a Escola Inglesa (Klein, Winnicott, Bion, Fairbairn), a Ego psychology (A. Freud, Hartmann, Kris, Loewenstein), a Escola Francesa (Lacan e seus discípulos), entre outras. Assim, nesta pesquisa considerou-se pertinente levantar a diversidade do campo.

Em relação à produção de conhecimento científico, este pode ser reconhecido por meio de sua sistematização no rigor dos critérios reconhecidos pela ciência. Assim sendo, faz parte da produção de conhecimento sua divulgação em meios confiáveis para que a informação seja socializada (Sampaio, 2008; Volpato, 2003). Sob esse aspecto, é fato que a produção de conhecimento em psicanálise e sua respectiva divulgação ainda caminha a passos lentos, ainda que a Revista de Psicanálise tenha sido a primeira revista de psicologia publicada no Brasil, no ano de 1928. Na ocasião, Durval Marcondes, após enviar uma carta a Freud, recebeu uma resposta na qual este expressava a ciência acerca do fato (Conselho Regional de Psicologia de São Paulo, 2009; Suehiro, Cunha, Oliveira \& Pacanaro, 2007).

Poucas pesquisas até o presente momento trataram do tema produção científica no contexto psicanalítico em âmbito nacional. Dentre os estudos realizados com esse foco, pode-se citar Yoshida, Santeiro, Santeiro e Rocha (2005), que fizeram um levantamento da produção científica nacional e estrangeira acerca das psicoterapias breves psicodinâmicas no período de 1980 a 2003. Uma outra investigação abrangendo o tema foi realizada por Ferreira e Yoshida (2004), que levantaram a produção científica de 1990 a 2000 sobre psicoterapias breves no Brasil e demais países latino-americanos. Uma das conclusões desses estudos é que em âmbito internacional a produção sobre o tema é muito mais expressiva. Ressalte-se que ambos os estudos, embora tenham um foco psicanalítico, centraram sua investigação sobre uma técnica específica, a saber, a psicoterapia breve psicodinâmica ao passo que o presente trabalho preocupou-se mais em traçar um panorama do campo baseado na teoria psicanalítica, abrangendo, portanto, as diferentes técnicas e orientações que nela se fundamentam.

A escassez de trabalhos que busquem proporcionar um panorama mais geral da produção científica no contexto psicanalítico é fato. Que o campo psicanalítico apresente-se de forma plural e heteróclita, não deveria servir para desencorajar a análise da produção bibliográfica da área. A sua multiplicidade convida justamente a um mapeamento dessas diferenças, a fim de não só visualizar-se um panorama da diversidade do campo como também se avaliar suas tendências, tensões e eventuais diálogos. Além disso, é oportuno constatar de que modo essas diferenças se configuram no Brasil, destacando as particularidades da área.

O presente estudo buscará refletir sobre as tendências de publicações realizadas no campo da produção científica em clínica psicanalítica, realizando um levantamento dos posicionamentos, bem como uma articulação entre as perspectivas teóricas de atuação nesse contexto. Com esse pressuposto, serão mapeados e discutidos, do ponto de vista metacientífico, alguns indicadores da produção e divulgação do conhecimento nessa área. Portanto, levantar a produção científica da área psicanalítica foi o objetivo deste estudo.

\section{Método}

Fontes

Foram analisados seis periódicos científicos na área de psicologia, cujo critério de escolha foi o fato de o periódico ser avaliado pela CAPES, tendo sua coleção disponível on-line. Também se primou por revistas que fossem representativas de diferentes estados brasileiros. As revistas analisadas foram: Psicologia: Reflexão e Crítica, Psicologia: Teoria e Pesquisa, Psicologia em Estudo (Maringá), PsicoUSF, Estudos de Psicologia (Natal) e Agora - Estudos em Teoria Psicanalítica, tratando-se esta última de uma publicação especializada em psicanálise.

\section{Procedimento}

Nessas revistas buscou-se avaliar somente os resumos dos artigos que focaram a psicanálise. Como o foco era a análise de artigos sobre assuntos da área da psicanálise, o critério de inclusão de um artigo na categoria psicanálise baseou-se na menção explícita a conceitos e/ou obras e/ou autores, inequivocamente oriundos do referencial psicanalítico.

Para tanto, estabeleceu-se um período de 8 anos (2002-2009) para a realização da análise, sendo que no ano de 2009 somente analisou-se o primeiro número publicado, pois nem todos os periódicos haviam disponibilizado os demais números. O total de artigos levantados foi 1.728, dos quais somente 229 tratavam-se especificamente de publicações relacionadas ao tema analisado. Foi feito um levantamento da região brasileira da qual o trabalho foi originário.

Os critérios definidos para a meta-análise dos periódicos foram baseados em Witter (1999). Para tanto, foram estabelecidos os seguintes itens: (a) Autoria, identificando-se a natureza (individual ou múltipla), o gênero dos autores, a proveniência das 
instituições dos autores (pública, privada, ambas); (b) Temática, analisando-se a quantidade e a distribuição por temas e teóricos da psicanálise; (c) Discurso, avaliando-se a natureza do trabalho (teórico ou prático), bem como as palavras contidas no seu título, (d) Análise dos recursos, para aqueles trabalhos nos quais houve coleta de dados, realizando-se a classificação do tipo de instrumentos empregados nas avaliações, bem como se elencando os instrumentos utilizados.

\section{Resultados}

Os dados foram organizados em planilha e submetidos à estatística descritiva. No que se refere à quantidade de volumes, números e o total de artigos publicados, a Tabela 1 apresenta o universo geral das publicações e a respectiva média de publicações gerais por ano e número. Cabe esclarecer que em todas as revistas foi analisado apenas o primeiro número publicado no ano de 2009, pois esse número já havia sido disponibilizado, enquanto nem todas tinham publicado os demais números.

Tabela 1 - Distribuição geral da quantidade de números, total de artigos publicados e média de publicações ano por número

\begin{tabular}{cccc}
\hline Ano & $\begin{array}{c}\text { Quantidade de } \\
\text { números }\end{array}$ & Total ano & $\begin{array}{c}\text { M de publicação } \\
\text { ano por número }\end{array}$ \\
\hline 2002 & 14 & 212 & 15,1 \\
2003 & 16 & 219 & 13,7 \\
2004 & 16 & 216 & 13,6 \\
2005 & 16 & 233 & 14,5 \\
2006 & 16 & 239 & 14,9 \\
2007 & 18 & 264 & 14,6 \\
2008 & 17 & 260 & 15,3 \\
2009 & 6 & 85 & 14,1 \\
\hline
\end{tabular}

Os dados apresentados (Tabela 1) parecem indicar que houve um aumento da produção científica no período analisado, havendo um aumento significativo das publicações a partir do ano 2005. Todavia, há que se ponderar que em 2009 a produção não correspondeu ao ano completo.
A Tabela 2 apresenta os dados relativos à publicação sobre psicanálise em cada periódico analisado, levando-se em consideração o montante geral de artigos por periódicos. É importante mencionar que, no caso da revista Psico-USF, no ano de 2002 somente foi disponibilizado o número 2.

Tabela 2 - Distribuição da publicação sobre psicanálise por periódico científico

\begin{tabular}{|c|c|c|c|c|c|c|}
\hline Ano & Agora & $\begin{array}{c}\text { Estudos de } \\
\text { Psicologia } \\
\text { (Natal) }\end{array}$ & $\begin{array}{l}\text { Psicologia: } \\
\text { Reflexão e } \\
\text { Crítica }\end{array}$ & $\begin{array}{c}\text { Psicologia em } \\
\text { Estudo } \\
\text { (Maringá) }\end{array}$ & Psico-USF & $\begin{array}{c}\text { Psicologia: Teoria e } \\
\text { Pesquisa }\end{array}$ \\
\hline 2002 & 18 & 46 & 61 & 29 & 22 & 36 \\
\hline 2003 & 16 & 53 & 56 & 42 & 21 & 31 \\
\hline 2004 & 14 & 58 & 44 & 48 & 22 & 30 \\
\hline 2005 & 16 & 47 & 53 & 54 & 22 & 41 \\
\hline 2006 & 16 & 36 & 60 & 62 & 26 & 39 \\
\hline 2007 & 17 & 31 & 59 & 64 & 32 & 61 \\
\hline 2008 & 20 & 30 & 61 & 60 & 28 & 61 \\
\hline 2009 & 9 & 9 & 19 & 22 & 11 & 15 \\
\hline $\begin{array}{c}\text { Total de } \\
\text { artigos }\end{array}$ & 126 & 310 & 413 & 381 & 184 & 314 \\
\hline $\begin{array}{l}\text { Artigos que } \\
\text { envolveram } \\
\text { psicanálise }\end{array}$ & 126 & 22 & 15 & 25 & 15 & 26 \\
\hline
\end{tabular}


Foi possível concluir, pela Tabela 2, que 229 artigos corresponderam ao montante de trabalhos que focaram a psicanálise. Embora a revista Psicologia: Reflexão e Crítica tenha obtido um maior número de publicações (n=413), foi a revista Ágora que, por ser especializada em publicações da psicanálise, teve sua totalidade de artigos $(\mathrm{n}=126)$ focados na psicanálise. Depois da Agora, as maiores ocorrências de artigos de psicanálise estavam na Psicologia: Teoria e Pesquisa $(\mathrm{n}=26)$, Psicologia em Estudo - Maringá $(\mathrm{n}=25)$ e Estudos de Psicologia - Natal $(\mathrm{n}=22)$.

Buscou-se averiguar as produções na área considerando as regiões brasileiras e também a proveniência internacional. $\mathrm{Na}$ Tabela 3 é possível constatar a frequência de artigos que focaram a psicanálise.

Tabela 3 - Distribuição da frequência dos artigos que focaram a psicanálise considerando a região brasileira $(\mathrm{n}=229)$

\begin{tabular}{lcc}
\hline Região & F & $\%$ \\
\hline Sul & 28 & 12,2 \\
Sudeste & 140 & 61,1 \\
Norte & 3 & 1,3 \\
Nordeste & 8 & 3,5 \\
Centro-oeste & 10 & 4,4 \\
Parceria entre estados & 4 & 1,7 \\
Internacional & 36 & 15,7 \\
\hline
\end{tabular}

Ao que parece, a região sudeste foi a que apresentou maior incidência $(n=140)$ de publicações voltadas à psicanálise. Poucas $(\mathrm{n}=4)$ foram as parcerias entre instituições de diferentes estados e quase inexpressiva $(\mathrm{n}=3)$ foi a ocorrência de trabalhos da região norte.

No que concerne à autoria, evidenciou-se que $69,4 \% \quad(n=159)$ das produções eram de natureza individual e 30,6\% ( $n=70)$ envolviam autoria múltipla. $O$ percentual do gênero masculino representando a autoria de trabalho foi de $31,9 \%(\mathrm{n}=73)$ e do feminino $53,3 \%(n=122)$, sendo $14,8 \%(n=34)$ o percentual de trabalhos que tiveram parceria entre homens $\mathrm{e}$ mulheres. As pesquisas provenientes de autores de instituições públicas representaram 58,1\% ( $\mathrm{n}=133)$, enquanto $28,4 \%(n=65)$ foi o percentual de pesquisas de instituições privadas, sendo também possível observar a parceria entre autores de universidades públicas e privadas $(13,5 \%, \mathrm{n}=31)$.
No que tange à análise da temática, buscou-se averiguar a quantidade e a distribuição por temas (Tabela 4) e por teóricos da psicanálise estudados (Tabela 5). Vale esclarecer que a classificação somente foi possível quando a informação estava explícita no resumo do artigo. O critério para atribuir o artigo a determinado autor é que no resumo tenha sido feita a menção explícita ao nome do autor, ou uma declaração explícita de que o trabalho foi feito a partir de seu referencial de leitura. Dessa forma, entrou na rubrica a categoria "não deixa claro/não se aplica", referente a trabalhos que, apesar de terem deixado clara sua pertinência à psicanálise, não evidenciaram no resumo de qual autor específico eram tributários, seja em virtude de uma insuficiência do resumo (não deixa claro), seja porque não seria pertinente a informação (não se aplica).

Tabela 4 - Distribuição da frequência dos artigos considerando o tema pesquisado $(n=229)$

\begin{tabular}{lcc}
\hline Categorias & $\mathrm{F}$ & $\%$ \\
\hline Estudo de casos & 9 & 3,9 \\
Estudo de casos clínicos & 11 & 4,8 \\
Psicanálise e outros saberes & 56 & 24,4 \\
Análise de construto psicanalítico & 102 & 44,5 \\
Estudos de/com testes psicológicos & 19 & 8,3 \\
Análise da cultura a partir da psicanálise & 21 & 9,2 \\
História da psicanálise & 3 & 1,3 \\
Análise da produção & 1 & 0,4 \\
Outros & 7 & 3,1 \\
\hline
\end{tabular}


A análise de construto psicanalítico foi a categoria de maior incidência de artigos $(n=102)$.
Apenas um trabalho focou a análise da produção sobre um construto específico da clínica psicanalítica.

Tabela 5 - Distribuição da frequência dos artigos considerando o teórico estudado ( $\mathrm{n}=229)$

\begin{tabular}{lcc}
\hline Teóricos & F & $\%$ \\
\hline Freud & 66 & 28,9 \\
Lacan & 25 & 10,9 \\
Freud e Klein & 1 & 0,4 \\
Ferenczi & 1 & 0,4 \\
Freud e Ferenczi & 3 & 1,3 \\
Lacan e Ferenczi & 1 & 0,4 \\
Freud e Lacan & 22 & 9,7 \\
Freud, Lacan e Miller & 1 & 0,4 \\
Freud e Karl Abraham & 1 & 0,4 \\
Freud, M. Schneider e P. Fédida & 1 & 0,4 \\
Freud, Winnicott e J. Chasseguet-Smirgel & 1 & 0,4 \\
Winnicott & 4 & 1,8 \\
Lacan e Winnicott & 1 & 0,4 \\
M. Balint & 1 & 0,4 \\
J. D. Nasio, J. Bergés e G. Balbo & 1 & 0,4 \\
Freud e Laplanche & 1 & 0,4 \\
Laplanche & 1 & 0,4 \\
Laplanche e J. André & 1 & 0,4 \\
J. Bowlby & 3 & 1,3 \\
J. Kestenberg & 2 & 1,0 \\
Não deixa claro/não se aplica & 91 & 39,8 \\
\hline
\end{tabular}

Os dados da Tabela 5 destacam a maior incidência de trabalhos utilizando Freud como referencial, sendo este seguido por Lacan. Esses dados já eram esperados.

$\mathrm{Na}$ análise do discurso, no tocante à natureza do trabalho, observou-se que $79,5 \%(n=182)$ eram produções que focaram a pesquisa teórica, 20,5\% $(\mathrm{n}=47)$ das quais primaram pela realização de pesquisas práticas. As palavras contidas no título do trabalho podem ser vistas na Tabela 6 .

Tabela 6 - Análise do número de palavras contidas nos títulos dos artigos analisados ( $\mathrm{n}=229)$

\begin{tabular}{lcc}
\hline $\begin{array}{l}\text { Categorias do número } \\
\text { de palavras }\end{array}$ & F & $\%$ \\
\hline 2 a 7 & 70 & 30,5 \\
8 a 10 & 78 & 34,1 \\
11 a 13 & 49 & 21,4 \\
14 ou mais & 32 & 14 \\
\hline
\end{tabular}

De um modo geral, as revistas recomendam que os títulos tenham em torno de 10 a 12 palavras. Com os dados pode-se notar que a maior parte $(\mathrm{n}=127)$ apresentou de 8 a 13 palavras.

Finalmente, foi realizada uma análise dos recursos, sendo feita assim a classificação do tipo de recurso empregado nos trabalhos que utilizaram esses materiais em seus estudos, conforme consta na Tabela 7. A relação dos instrumentos estudados pode ser vista na Figura 1. 
Tabela 7 - Análise dos recursos empregados em artigos práticos $(n=29)$

\begin{tabular}{lcc}
\hline Tipos de recursos & F & $\%$ \\
\hline Recursos fatoriais & 1 & 3,4 \\
Questionários & 5 & 17,3 \\
Entrevista & 1 & 3,4 \\
Roteiros & 1 & 3,4 \\
Observação & 1 & 3,4 \\
Técnica/teste projetivos & 15 & 51,7 \\
Utiliza vários recursos ao mesmo tempo & 5 & 17,3 \\
\hline
\end{tabular}

Constatou-se que dos 47 trabalhos práticos, 29 recorreram aos recursos de medida. Dentre todos os recursos, a técnica projetiva foi privilegiada na maior parte dos trabalhos.

\begin{tabular}{|l|}
\hline \\
\hline Bateria de Grafismo de Hammer \\
Rorschach \\
Desenho história \\
Pfister \\
Desenho da Figura Humana \\
BBT \\
Zulliger \\
Teste do Desenho da Família \\
Teste das Fábulas \\
Wartegg
\end{tabular}

Figura 1 - Relação dos instrumentos utilizados nos estudos

Cabe esclarecer que os instrumentos constantes na Figura 1 foram apenas levantados. Não houve a preocupação de averiguar a frequência com que apareceram, portanto, todos que apareceram foram citados na figura.

\section{Discussão e conclusão}

Há uma carência de investigações que primem pela análise da produção científica no campo psicanalítico. Esse fato deve ser considerado, pois a falta de fundamentos mais consolidados impõe um limite para a discussão dos resultados aqui obtidos. Se por um lado essa limitação existe, por outro também é imprescindível dizer que a realização da presente investigação nos possibilita traçar um panorama da produção científica da área no Brasil, de modo a respaldar investigações futuras sobre o tema. Aliás, o número restrito ou a inexistência de estudos como o que aqui se propõe já seria uma justificativa para sua execução.

A análise da produção científica oferece um parâmetro de qualidade acerca do conhecimento produzido. As bases de dados científicas fornecem um meio seguro de se obter informação científica. Embora os periódicos disponíveis nessas bases sejam avaliados pelos critérios estabelecidos no Qualis/CAPES, ainda há muitas discussões sobre a aplicabilidade destes na avaliação das revistas brasileiras de psicologia. Apesar das críticas, é fato que a qualidade da produção brasileira em psicologia caminha para um parâmetro mais próximo daquele adotado em âmbito internacional.

Parece que a produção na área psicanalítica obedece à tendência geral da área de psicologia de um predomínio de mulheres em publicações científicas. Também foi fato a maior incidência de instituições públicas e a proveniência da região sudeste, o que corrobora o quadro atual, no qual a região sudeste agrega grande parte dos bons programas de PósGraduação stricto sensu, que por sua vez, também recebem mais investimentos dos órgãos de fomento.

Ao considerar o universo geral de publicações $(n=1.728)$, poucos foram os trabalhos que focaram a psicanálise. Desse modo, questionam-se as razões para que esse fato tenha ocorrido. Uma hipótese que poderia ser aventada seria o modo de divulgação desse conhecimento, que muitas vezes ocorre em escolas de psicanálise. Com isso fomenta-se que haveria grande número de bons trabalhos que ainda não foram divulgados em periódicos científicos indexados. A abrangência dessa informação privilegia apenas alguns grupos, que teriam acesso a esse conhecimento. Nessa direção, Sampaio (2008) e Volpato (2003) arrematam a 
necessidade e importância da socialização do conhecimento científico. Nessa direção, pode-se fomentar que, embora tenha havido um crescimento dos periódicos de psicologia em bases como o Index Psi (Costa \& Yamamoto, 2008), as conclusões obtidas por Ferreira e Yoshida (2004) e Yoshida e cols. (2005) revelam que esse crescimento ainda é pequeno, especialmente quando se considera a produção científica internacional.

Outro aspecto a ser considerado é a natureza de grande parte dos trabalhos na área psicanalítica, que parecem ser, em sua maior parte, teóricos. Tal fato poderia gerar certos obstáculos para a publicação desse material, uma vez que a política editorial de algumas revistas privilegia trabalhos de cunho prático. Isso não significa necessariamente que as revistas analisadas tenham essa prática.

No tocante aos trabalhos práticos, observouse que poucos foram aqueles que empregaram recursos de medida em seu levantamento de dados. A técnica projetiva foi o recurso que apresentou maior incidência de utilização. Contudo, pode-se considerar quase inexpressivo o número de artigos da área que recorre a tais técnicas, sobretudo, quando se considera o cômputo geral das publicações na área.

Houve um número elevado de artigos nos quais não foi possível detectar a filiação teórica no resumo. Essa poderia ser considerada uma limitação da presente pesquisa. Assim, em um estudo futuro seria necessária a leitura na íntegra, do material. Ressalte-se, no entanto, a relevância de se informar no resumo o referencial utilizado em trabalhos da área psicanalítica, especialmente aqueles de cunho teórico, uma vez que o resumo é uma ferramenta importante para que outros pesquisadores possam recuperar a informação desejada, constituindo um dos primeiros contatos que se tem com o artigo.

A maior incidência de Freud na categoria autores era esperada, uma vez que ele foi o fundador da psicanálise. Notou-se também a presença relevante da adoção do referencial lacaniano nos trabalhos, o que sinaliza para sua marcante presença no cenário acadêmico. Cabe mencionar que havia uma expectativa de que os representantes da Escola Inglesa exibissem uma participação expressiva, o que, contudo, não se confirmou nas revistas analisadas. Isso não significa que em outros periódicos essa situação se repetiria.

Em síntese, argumenta-se que, se é digno de nota o aumento gradativo da produção científica em psicologia, também ficou evidente, neste artigo, que a área da psicanálise, embora já consolidada no Brasil, necessita ampliar a divulgação de suas produções. Apesar de este estudo apresentar a limitação de trabalhar com a análise de seis periódicos, também é fato que as revistas analisadas apresentam reconhecida inserção na psicologia e são representativas de diferentes estados brasileiros. Ainda assim, para se chegar a um dado mais fidedigno da produção em psicanálise, seria pertinente sugerir a realização de novos estudos que envolvessem outros periódicos.

\section{Referências}

Barbosa, A. J. G. \& Moreira, P. S. (2009). Deficiência mental e inclusão escolar: produção científica em Educação e Psicologia. Revista Brasileira de Educação Especial, 15(2), 337-352.

Berberian, A. P., Kruger, S., Guarinello, A. C. \& Massi, G. A. A. (2009). A produção do conhecimento em fonoaudiologia em comunicação suplementar e/ou alternativa: análise de periódicos. Revista CEFAC, 11 (supl. 2), 258-266.

Cheniaux, E. A. (2006). Psicanálise se reaproximando das neurociências: um retorno a Freud. Em S. Alberti \& A. C. Figueiredo (Orgs.). Psicanálise e saúde mental: uma aposta (pp. 101-110). Rio de Janeiro: Companhia de Freud.

Conselho Regional de Psicologia de São Paulo (2009). Linha do tempo. Obtido em 6 de dezembro de 2009 do World Wide Web: $<$ http://www.crpsp.org.br/linha $>$.

Costa, A. L. F. (2006). Publicações e avaliações de periódicos cientificos: paradoxos de classificação QUALIS em psicologia. Dissertação de Mestrado, Universidade Federal do Rio Grande do Norte, Rio Grande do Norte.

Costa, A. L. F. \& Yamamoto, O. H. (2008). Publicação e avaliação de periódicos científicos: paradoxos da avaliação Qualis de Psicologia. Psicologia em Estudo, Maringá, 13(1), 13-24.

Ferreira, S. N. \& Yoshida, E. M. P. (2004). Produção científica sobre psicoterapias breves no Brasil e demais países latino-americanos (1990-2000). Estudos de Psicologia, 9(3), 523-531.

Freire, A. B. \& Lustoza, R. Z. (2005). A psicanálise entre a razão e a causa. Pulsional Revista de Psicanálise, 23(184), 28-39.

Lustoza, R. Z. (2007). O problema da causalidade psíquica na psicanálise. Tese de Doutorado, Universidade Federal do Rio de Janeiro, Rio de Janeiro.

Monzani, L. R. (1989). Freud: o movimento de um pensamento. Campinas: Editora da Unicamp. 
Oliveira Filho, R. S., Hochman, B. N. F. X. \& Ferreira, L. M. (2005). Fomento à publicação científica e proteção do conhecimento científico. Acta Cirúrgica Brasileira, 20 (2 suplemento), 35-39.

Sampaio, M. I. C. (2008). Citações a periódicos na produção científica de Psicologia. Psicologia: Ciência e Profissão, 28(3), 452-465.

Simanke, R. T. (2009). A psicanálise freudiana e a dualidade entre naturais e ciências humanas. Scientiae Studia, 7(2), 221-235.

Soares, A. B., Victória, M. S., Cavalieri, A. M. A. P., \& Bottino, A. G. (2006). A psicologia divulgada através de periódicos científicos indexados e através de revistas de grande circulação do Brasil: um estudo preliminar. Psicologia América Latina [online]. n. 7. Obtido em 10 de dezembro de 2009 na World Wide Web: <http://pepsic.bvspsi.org.br/scielo.php>.

Souza Filho, M. L., Belo, R. \& Gouveia, V. V. (2006). Testes Psicológicos: análise da produção científica no período de 2000-2004. Psicologia: Ciência e Profissão, 26(3), 478-489.

Suehiro, A. C. B., Cunha, N. B. \& Oliveira, E. Z. P. Pacanaro, S. V. (2007). Produção científica da revista Psico-USF de 1996 a 2006. PsicoUSF, 12(2), 327-334.
Volpato, G. L. (2003). Publicaşão cientifica (2 $2^{\mathrm{a}}$ ed.). Botucatu, SP: Tipomic.

Witter, G. P. (1999). Metaciência e leitura. Em G.P. Witter (Org.). Leitura: textos e pesquisas (pp. 13-22). Campinas: Alínea.

Witter, G. P. (2005). Metaciência e psicologia. Campinas: Alínea.

Yamamoto, O. H., Souza, C., \& Yamamoto, M. E. (1999). A produção científica na psicologia: uma análise dos periódicos brasileiros no período de 1990-1997. Psicologia: Reflexão e Crítica, 12(2), 549565.

Yamamoto, O. H., Menandro, P. R. M, Koller, S. H., LoBianco, A. C., Hutz, C., Bueno, J. L. C., \& Guedes, M. C. (2002). Avaliação de periódicos científicos brasileiros na área da psicologia. Ciência da Informação, 31(2), 163-177.

Yoshida, E. M. P., Santeiro, T. V., Santeiro, F. R. M. \& Rocha, G. M. A. (2005). Psicoterapias breves psicodinâmicas: características da produção científica nacional e estrangeira (1980/2003). PsicoUSF, 10(1), 51-59.
Recebido em janeiro de 2010

Reformulado em abril de 2010 Aprovado em maio de 2010

Sobre as autoras:

Rosane Zétola Lustoza é psicóloga, mestre em Teoria Psicanalítica pela UFRJ, doutora em Teoria Psicanalítica pela UFRJ e professora adjunta do Curso de Psicologia/PPSIC da Universidade Estadual de Londrina.

Katya Luciane de Oliveira é psicóloga, mestre em Psicologia pelo Programa de Pós-Graduação Stricto Sensu em Psicologia da Universidade São Francisco, doutora em Psicologia, Desenvolvimento Humano e Educação pela Faculdade de Educação da Unicamp e professora adjunta doutora do Curso de Psicologia/PPSIC e do Mestrado em Educação da Universidade Estadual de Londrina.

Bianca Novaes de Mello é psicóloga, mestre em Teoria Psicanalítica pela UFRJ e doutoranda da Psicologia Clínica pela Pontifícia Universidade Católica do Rio de Janeiro. 